\title{
Linear stability of thermocapillary liquid layers of a shear-thinning fluid
}

Kai-Xin Hu, Meng He, Qi-Sheng Chen, and Rong Liu

Citation: Physics of Fluids 29, 073101 (2017);

View online: https://doi.org/10.1063/1.4994596

View Table of Contents: http://aip.scitation.org/toc/phf/29/7

Published by the American Institute of Physics

\section{Articles you may be interested in}

Marangoni effects on a thin liquid film coating a sphere with axial or radial thermal gradients Physics of Fluids 29, 072106 (2017); 10.1063/1.4991580

Liquid jet leaping from a free surface

Physics of Fluids 29, 071702 (2017); 10.1063/1.4994601

Floquet analysis of spatially periodic thermocapillary convection in a low-Prandtl-number liquid bridge

Physics of Fluids 29, 074104 (2017); 10.1063/1.4993466

Three-dimensional dynamics of thin liquid films on vertical cylinders with Marangoni effect

Physics of Fluids 29, 011701 (2017); 10.1063/1.4974076

The linear stability of Hunt-Rayleigh-Bénard flow

Physics of Fluids 29, 064103 (2017); 10.1063/1.4984842

Effect of uniform electric field on the drop deformation in simple shear flow and emulsion shear rheology

Physics of Fluids 29, 072109 (2017); 10.1063/1.4995473

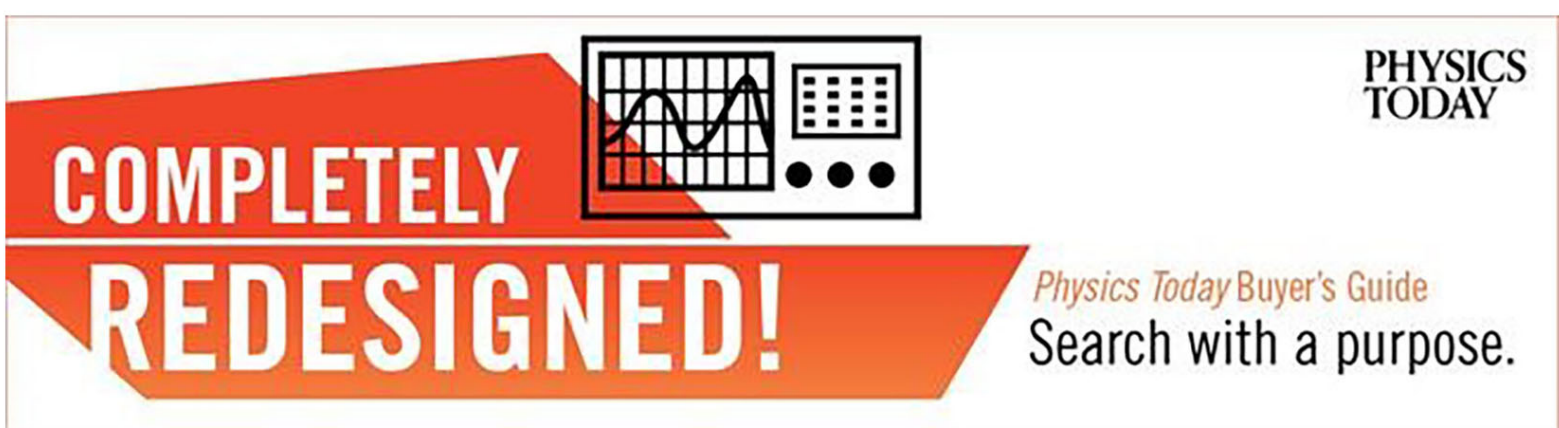




\title{
Linear stability of thermocapillary liquid layers of a shear-thinning fluid
}

\author{
Kai-Xin Hu, ${ }^{1, a)}$ Meng He, ${ }^{2}$ Qi-Sheng Chen, ${ }^{2}$ and Rong Liu ${ }^{3}$ \\ ${ }^{1}$ School of Mechanical Engineering and Mechanics, Ningbo University, Ningbo, Zhejiang 315211, China \\ ${ }^{2}$ National Microgravity Laboratory, Institute of Mechanics, Chinese Academy of Sciences, \\ Beijing 100190, China \\ ${ }^{3}$ School of Mechanical and Electrical Engineering, Guilin University of Electronic Technology, \\ Guilin 541004, China
}

(Received 7 April 2017; accepted 3 July 2017; published online 21 July 2017)

\begin{abstract}
The linear stability analysis has been performed for the thermocapillary liquid layers of a shearthinning fluid. The Carreau fluid model is applied to describe the rheological property. The critical parameters are determined as a function of Prandtl number $(P r)$, degree of shear-thinning, and gravity level. For linear flow, the shear-thinning effect is destabilizing for small and moderate $\operatorname{Pr}$ but increases the stability slightly for large $P r$. For return flow, the perturbation kinetic energy concentrates near the surface, and the flow is stabilized when the surface viscosity is used. The streamwise wave is excited at large $\mathrm{Pr}$, and a new mechanism is found at moderate $\mathrm{Pr}$, where the hot spots appear at the bottom of the layer. In the presence of gravity, the viscosity stratification is enhanced and more kinds of different modes are excited. The preferred mode changes to downstream at large $P r$ while the gravity becomes an important perturbation energy source at small Pr. The shear-thinning effect for the instability mechanism is discussed and the comparisons are made with channel flows. Published by AIP Publishing. [http://dx.doi.org/10.1063/1.4994596]
\end{abstract}

\section{INTRODUCTION}

Thermocapillary flow is the fluid motion driven by the temperature-induced surface tension gradient. As the flow stability is crucial in many applications, such as crystal growth ${ }^{1}$ and fusion welding, ${ }^{2}$ the instability of thermocapillary flow has been studied over the years. The theoretical and experimental studies have been reviewed by Davis ${ }^{3}$ and Schatz and Neitzel. ${ }^{4}$ The thermocapillary flows of polymer liquids appear in many practical applications, such as film coating,,${ }^{5,6}$ drying of polymer solution, ${ }^{7-9}$ deliberate patterning of polymer, ${ }^{10}$ lithography, ${ }^{11,12}$ inkjet printing, ${ }^{13}$ and polymer processing in microgravity. ${ }^{14}$

It is worth noting that the rheological property of polymer liquids varies considerably from Newtonian fluid. The shear thinning combined with elastic effects is produced in the polymer processing, which have great impacts on the flow. The effect of viscoelasticity on the stability of thermocapillary liquid layers has been investigated in some previous studies, ${ }^{15-17}$ which show that the instability can be markedly affected by elasticity.

A few papers have been devoted to the study of thermocapillary flows for shear-thinning fluid. The Marangoni convection in a shallow rectangular cavity of a power-law fluid has been investigated by Naimi, Hasnaoui, and Platten ${ }^{18}$ and Alloui and Vasseur. ${ }^{19}$ The shear-thinning effect on the fluid flow, temperature field, and heat transfer is discussed. Chen ${ }^{20}$ has examined the influence of Marangoni convection for a power-law liquid film on an unsteady stretching sheet. The effect of thermocapillary force for the velocity and

a) Email: hjhhkx@126.com temperature is demonstrated for several power-law indexes. However, the shear-thinning effect for the stability of thermocapillary flow has not been discussed thoroughly in previous studies.

The viscosity often varies in space in shear-thinning fluid flows. There are two ways in which the viscosity variation can affect the flow stability. The first is viscosity stratification. ${ }^{21}$ The instabilities in viscosity-stratified flow have been reviewed by Govindarajan and Sahu. ${ }^{22}$ The second is the viscosity disturbance, which yields an anisotropic disturbance stress tensor. ${ }^{23}$ Liu and $\mathrm{Liu}^{24}$ have examined the non-modal instability in plane Couette flow of a power-law fluid, which has no viscosity stratification. The results show that the shearthinning significantly increases the amplitude of response to external excitations and initial conditions. In common cases, there are both stratification and disturbance for the viscosity. The stability of shear-thinning fluids in channel flow has been examined by Nouar, Bottaro, and Brancher. ${ }^{23}$ The viscosity disturbance is accounted. The results show that viscous stratification can stabilize the flow when an appropriate viscosity scale is employed in the definition of the Reynolds number. Nouar and Frigaard ${ }^{25}$ have studied the stability of plane Couette-Poiseuille flow of shear-thinning fluid, which indicates that the effect of the shear thinning leads to a decrease in the phase velocity of the traveling waves and an increase in stability. Thus, one would expect that the shear-thinning effect may have a great impact on the stability of thermocapillary flow.

In the present work, the instability of thermocapillary convection for a shear-thinning fluid is investigated by threedimensional linear stability analysis. The model of thermocapillary liquid layer is considered, while the Carreau fluid is applied to model the polymer liquid. The flows with and 
without viscosity stratification are discussed, and the effect of gravity is demonstrated.

The paper is organized as follows. In Sec. II, the physical models and mathematical formulation are presented. The property of basic flow is discussed and the governing equations are derived. Then in Sec. III, numerical results for linear flow, return flow, and the flow under gravity are obtained, respectively. The perturbation flow field is displayed and the energy mechanism is studied. After that, the instability mechanism is discussed in Sec. IV. Finally, we summarize the results and present the conclusions in Sec. V.

\section{PROBLEM FORMULATION}

We use the model of thermocapillary liquid layer proposed by Smith and Davis, ${ }^{26}$ where a fluid layer is above an infinite rigid plane and a temperature gradient is imposed on its free surface (see Fig. 1). The convection is driven by the thermocapillary force while the surface tension is large enough to keep the surface flat. $x, y$, and $z$ are the streamwise, spanwise, and wall-normal direction, respectively. This model has been widely adopted in the theoretical study of thermocapillary convection and has proven capable of predicting the oblique hydrothermal waves observed in the experiment ${ }^{27}$ and numerical simulation. ${ }^{28}$ The linear flow and return flow are considered, which will be introduced later. The magnitude of the surface deformation can be measured by the capillary number, ${ }^{26,27} \mathrm{Ca}=\hat{\mu}_{0} \hat{U}_{0} / \hat{\sigma}$, where $\hat{\mu}_{0}, \hat{U}_{0}$, and $\hat{\sigma}$ are the characteristic viscosity, velocity, and surface tension, respectively. In some practical applications, such as liquid silicon ${ }^{26}$ and silicone oil, ${ }^{27} O(\mathrm{Ca}) \approx 10^{-3} \ll 1$. So the assumption of a non-deformable free surface is satisfied.

\section{A. Governing equations}

We choose the Carreau fluid model to describe the behavior of the shear-thinning fluid, as this model can be obtained from Lodge's molecular network theory ${ }^{29}$ and is sufficient to fit a wide variety of polymer liquids in the experiment. ${ }^{30}$ Its constitutive equation is

$$
\boldsymbol{\tau}=\hat{\mu}(\dot{\gamma}) \dot{\gamma},
$$

where $\boldsymbol{\tau}$ is the stress tensor, $\dot{\gamma}$ is the strain-rate tensor with the form $\dot{\gamma}=\nabla \mathbf{u}+\mathbf{u} \nabla$, $\mathbf{u}$ is the velocity, $\hat{\mu}$ is the viscosity that depends on the magnitude of the strain-rate tensor $\dot{\gamma}$ $=\left(\frac{1}{2} \dot{\gamma}: \dot{\gamma}\right)^{1 / 2}$,

$$
\hat{\mu}=\hat{\mu}_{\infty}+\left(\hat{\mu}_{0}-\hat{\mu}_{\infty}\right)\left[1+(\hat{\lambda} \dot{\gamma})^{2}\right]^{(n-1) / 2} .
$$

Here, $\hat{\mu}_{0}$ and $\hat{\mu}_{\infty}$ are the viscosities at zero and infinite shear rate $\left(\hat{\mu}_{0} \geq \hat{\mu}_{\infty}\right)$, respectively, the power-law index $n$ represents the degree of shear-thinning $(n \leq 1)$, and $\hat{\lambda}$ is the material time constant. It can be seen that the extent of shearthinning always increases with the increasing of $\hat{\lambda}$ or decreasing of $n$, and the Carreau fluid recovers Newtonian fluid when $n=1$.

The characteristic viscosity is chosen as $\hat{\mu}_{0}$, which is similar as that in Ref. 23. The dimensionless parameters are defined as follows: $R$ is the Reynolds number $R=\rho \hat{U}_{0} d / \hat{\mu}_{0}$, where $\rho$ is the fluid density, $\hat{U}_{0}$ is the characteristic velocity with the expression $\hat{U}_{0}=b \gamma d / \hat{\mu}_{0}$. Here, $b$ is the temperature gradient on the surface and $\gamma$ is the negative rate of change of surface tension with temperature. $M a$ is the Marangoni number defined as $M a=b \gamma d^{2} /\left(\hat{\mu}_{0} \chi\right)$, where $\chi$ is the thermal diffusivity. $M a$ and $R$ have the relation $M a$ $=R \cdot \operatorname{Pr}$, where $\operatorname{Pr}$ is the Prandtl number $\operatorname{Pr}=\hat{\mu}_{0} /(\rho \chi)$. In the presence of gravity, Boussinesq approximation is used. The fluid density is associated with the temperature $\rho$ $=\rho_{0}\left[1-a\left(\tilde{T}-\tilde{T}_{0}\right)\right]$, where $a$ is the thermal expansion coefficient, $\tilde{T}$ and $\tilde{T}_{0}$ are the temperature of fluid and the reference temperature, respectively. The gravity effect is measured by the dynamic Bond number $B o=\rho g a d^{2} / \gamma$ and $g$ is the gravitational acceleration.

Then, the dimensionless expression of viscosity $\mu$ has the form

$$
\mu=\mu_{\infty}+\left(1-\mu_{\infty}\right)\left[1+(\lambda \dot{\gamma})^{2}\right]^{(n-1) / 2},
$$

where $\mu_{\infty}=\hat{\mu}_{\infty} / \hat{\mu}_{0}, \lambda=\hat{\lambda} \hat{U}_{0} / d$. In the following, we restrict our attention to the case $\mu_{\infty}=0.01$. The dimensionless governing equations are given below, which are the continuity equation, the momentum equation, and the energy equation, respectively.

$$
\begin{aligned}
\nabla \cdot \mathbf{u} & =0, \\
R\left(\frac{\partial \mathbf{u}}{\partial t}+\mathbf{u} \cdot \nabla \mathbf{u}\right) & =-\nabla p+\nabla \cdot \boldsymbol{\tau}+B o \cdot T \mathbf{e}_{3}, \\
\frac{\partial T}{\partial t}+\mathbf{u} \cdot \nabla T & =\frac{1}{M a} \nabla^{2} T .
\end{aligned}
$$

Here $p$ and $T$ stand for the pressure and temperature, respectively. The viscous dissipation of fluid is ignored in (2.6). The boundary conditions are set in the following:

$$
\begin{aligned}
\mathbf{u} & =(u, v, w)=0, \frac{\partial T}{\partial z}=0, z=0, \\
\tau_{13}+\frac{\partial T}{\partial x} & =0, \tau_{23}+\frac{\partial T}{\partial y}=0, w=0, \\
-\frac{\partial T}{\partial z} & =B i \cdot\left(T-T_{\infty}\right)+\tilde{Q}, z=1 .
\end{aligned}
$$

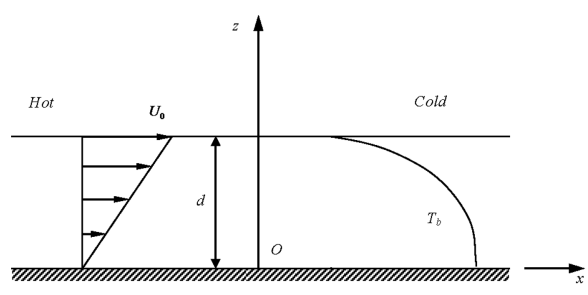

(a)

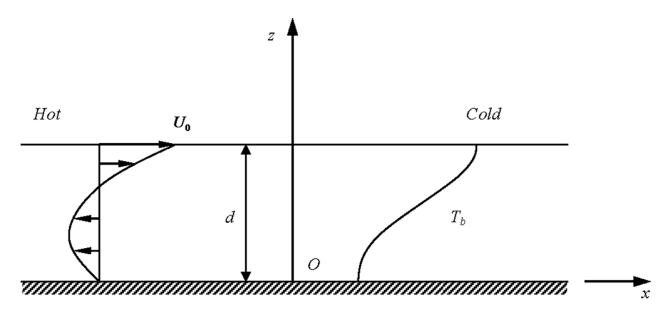

(b)
FIG. 1. Schematic of thermocapillary liquid layers: (a) linear flow and (b) return flow. Here, $d$ is the depth of the layer, $T_{b}$ is the temperature distribution in vertical direction, $U_{0}$ is the velocity field. 
Here, there is no slip and zero heat flux on the rigid plane. On the free surface, the stress on the surface is caused by the thermocapillary effect. $T_{\infty}$ is the temperature of the bounding gas far from the surface. $B i$ is the Biot number. As it always makes the flow more stable, ${ }^{26}$ for simplicity, we set it as zero. $\tilde{Q}$ is the imposed heat flux to the environment, which can be determined by the form of basic flow. ${ }^{26}$

We assume that the basic flow is parallel while its temperature is linear in $x$ as imposed plus a distribution in $z$ as following:

$$
\mathbf{u}=\left(U_{0}(z), 0,0\right), T_{0}(x, z)=-x+T_{b}(z) .
$$

Two kinds of flows are investigated. The first kind is the linear flow whose velocity is linear in $z$. The solution has the form as following. It is easy to find that this flow can only exist when $B o=0$.

$$
\mathbf{u}=(z, 0,0), T_{0}(x, z)=-x+M a \cdot \frac{1}{6}\left(1-z^{3}\right) .
$$

The second kind is the return flow, which has zero mass flux in the vertical section,

$$
\int_{0}^{1} U_{0}(z) \mathrm{d} z=0
$$

The analytical solution of return flow cannot be obtained for the Carreau model. However, it can be derived numerically. The details for the derivation are described in the Appendix. The distributions of basic velocity and temperature for return flow are displayed in Fig. 2. Here, $U_{0}$ is independent on $\mathrm{Pr}$, as it can be derived from the distribution of shear stress in (A4). The latter can be obtained with the boundary condition $\left.\tau_{13}\right|_{z=1}=1$ and the return flow condition (2.11), which are both independent on $\mathrm{Pr}$. However, the temperature of basic flow depends on $M a$, which can be seen from (A5). It can be seen in Fig. 2 that the gradients of velocity and temperature in vertical direction always increase with the extent of shearthinning (increasing of $\lambda$ or decreasing of $n$ ) and $B o$.

\section{B. Perturbation equations}

An infinitesimal perturbation in the normal mode form is added to the basic flow,

$$
\begin{gathered}
(\mathbf{u}, T, P, \boldsymbol{\tau})=\left(\mathbf{u}_{0}, T_{0}, P_{0}, \boldsymbol{\tau}_{0}\right)+\boldsymbol{\delta} \\
\boldsymbol{\delta}=(\widehat{u}, \widehat{v}, \widehat{w}, \widehat{T}, \widehat{P}, \widehat{\tau}) \exp [\sigma t+i(\alpha x+\beta y)] .
\end{gathered}
$$

The subscript 0 stands for the basic flow and hereafter, the variables without subscript 0 stand for the perturbation. $\sigma=\sigma_{r}$ $+\mathrm{i} \sigma_{i}$, where $\sigma_{r}$ and $\sigma_{i}$ are the growth rate and frequency, respectively. $\alpha$ and $\beta$ are the wave number in the $x$ and $y$ directions, respectively. The wave number and the propagation angle are defined as $k=\sqrt{\alpha^{2}+\beta^{2}}$ and $\phi=\tan ^{-1}(\beta / \alpha)$, respectively. Due to symmetry, we shall confine ourselves to the case of $\phi \in\left[0^{\circ}, 180^{\circ}\right]$.

As the viscosity depends on the shear rate [see (2.3)], the perturbation of strain-rate $\dot{\gamma}$ can lead to the viscosity perturbation $\mu^{\prime}$. Thus, it can be inferred from (2.1) that the perturbation stress $\boldsymbol{\tau}$ consists of two parts: the first is caused by the perturbation of strain-rate $\dot{\gamma}$ while the second is caused by the viscosity perturbation $\mu^{\prime}$,

$$
\boldsymbol{\tau}=\mu \dot{\gamma}+\mu^{\prime} \dot{\gamma}_{0}
$$

where $\dot{\gamma}_{0}$ is the strain-rate of the basic flow. In this paper,

$$
\dot{\gamma}_{0}=\left(\begin{array}{ccc}
0 & 0 & \dot{\gamma}_{0} \\
0 & 0 & 0 \\
\dot{\gamma}_{0} & 0 & 0
\end{array}\right), \dot{\gamma}_{0}=D U_{0}, D=\frac{\mathrm{d}}{\mathrm{d} z}
$$

Substituting (2.12a) and (2.12b) into (2.3) and (2.13), the linearized relation between the perturbation of stress and strain-rate is obtained as follows:

$$
\begin{aligned}
\widehat{\tau}= & \mu\left(\begin{array}{ccc}
2 \mathrm{i} \alpha \widehat{u} & \mathrm{i} \alpha \widehat{v}+i \beta \widehat{u} D \widehat{u}+\mathrm{i} \alpha \widehat{\mathrm{w}} \\
\mathrm{i} \alpha \widehat{v}+i \beta \widehat{u} & 2 \mathrm{i} \beta \widehat{v} & \mathrm{i} \beta \widehat{w}+D \widehat{v} \\
D \widehat{u}+i \alpha \widehat{w} & \mathrm{i} \beta \widehat{w}+D \widehat{v} & 2 D \widehat{w}
\end{array}\right) \\
+ & \frac{\mathrm{d} \mu}{\mathrm{d} \dot{\gamma}_{0}} \dot{\gamma}_{0}\left(\begin{array}{ccc}
0 & 0 D \widehat{u}+\mathrm{i} \alpha \widehat{w} \\
0 & 0 & 0 \\
D \widehat{u}+\mathrm{i} \alpha \widehat{w} & 0 & 0
\end{array}\right),
\end{aligned}
$$

where

$$
\frac{\mathrm{d} \mu}{\mathrm{d} \dot{\gamma}_{0}} \dot{\gamma}_{0}=\left(1-\mu_{\infty}\right)(n-1)\left[1+\left(\lambda \dot{\gamma}_{0}\right)^{2}\right]^{(n-3) / 2}\left(\lambda \dot{\gamma}_{0}\right)^{2}
$$

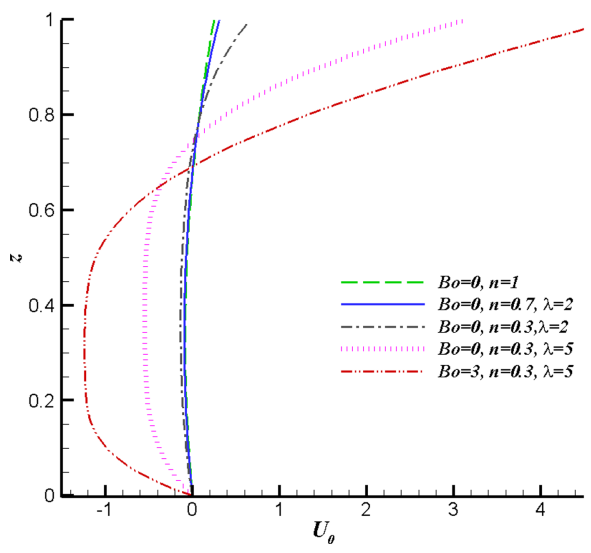

(a)

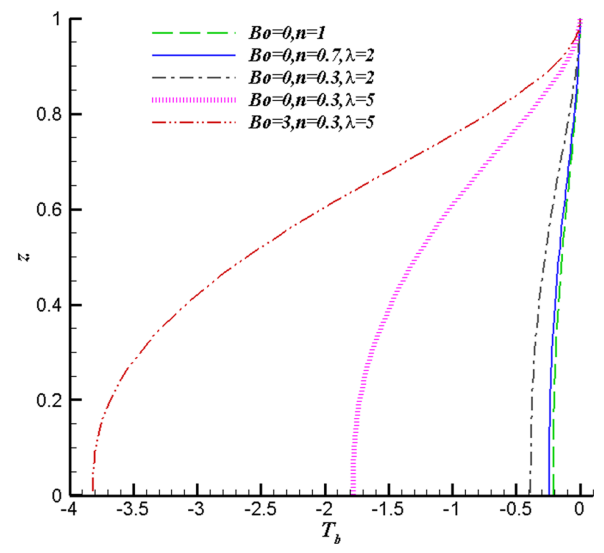

(b)
FIG. 2. The distributions of (a) basic velocity and (b) temperature at $M a=10$ for return flow. 


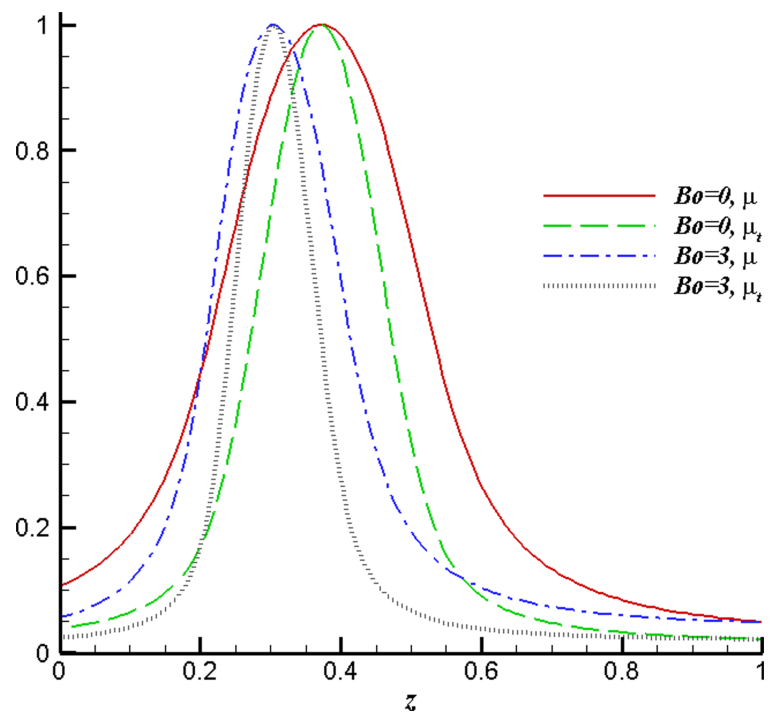

FIG. 3. The distributions of viscosity $\mu$ and tangent viscosity $\mu_{t}$ for return flow at $n=0.3, \lambda=5$.

Here, (2.16) is deduced from (2.3). The two parts in the right side of (2.15) correspond to those in (2.13), respectively. When $n=1$ (Carreau fluid degenerates to Newtonian fluid), the second part disappears [see (2.16)]. Then, (2.15) has the same form as that for Newtonian fluid, where the perturbation stress is proportional to the perturbation of strain-rate. When $n<1$, due to the second part, the viscosity corresponds to $\dot{\gamma}_{13}$ becomes

$$
\mu_{t}=\mu+\frac{\mathrm{d} \mu}{\mathrm{d} \dot{\gamma}_{0}} \dot{\gamma}_{0}
$$

However, the viscosity for other components of $\dot{\gamma}$ is $\mu$. In Ref. $23, \mu_{t}$ is termed as the tangent viscosity, as it is the slope of tangent line for the curve of shear stress versus shear rate. It can be seen from (2.16) that $\mu_{t} \leq \mu$ for a shear thinning fluid $(n<1)$.
The distributions of viscosity $\mu$ and tangent viscosity $\mu_{t}$ for the return flow are displayed in Fig. 3. Here, the distributions of viscosity $\mu$ and tangent viscosity $\mu_{t}$ are independent of $M a$ and $P r$, as both of them can be derived from the distribution of basic velocity $U_{0}$ [see (2.3) and (2.17)]. The latter is independent of $M a$ and $P r$. It can be seen that both $\mu$ and $\mu_{t}$ reach their minimum on the surface while the maximum appears when $\dot{\gamma}_{0}=0$. The maximum of $\mu$ is about 20 times larger than its minimum, while the corresponding value for $\mu_{t}$ is about 40 . The gravity does not change the values of $\mu$ and $\mu_{t}$ at $z=1$, which can be seen from (2.8) and (2.9) that

$$
\left.\left(\mu_{\infty}+\left(1-\mu_{\infty}\right)\left[1+\left(\lambda \dot{\gamma}_{0}\right)^{2}\right]^{(n-1) / 2}\right) \dot{\gamma}_{0}\right|_{z=1}=1
$$

When $n$ and $\lambda$ are fixed, the value of $\dot{\gamma}_{0}, \mu$, and $\mu_{t}$ can be obtained from the above equation. In contrast, the gravity decreases $\mu$ and $\mu_{t}$ at $z=0$. In the presence of gravity, the average of $\mu$ decreases from $0.378(B o=0)$ to 0.270 ( $B o$ $=3$ ) in Fig. 3, while the case for $\mu_{t}$ is similar. This indicates that the extent of shear-thinning is enhanced by the gravity.

The boundary conditions of perturbation flow are determined as follows:

$$
\widehat{u}=\widehat{v}=\widehat{w}=\frac{\partial \widehat{T}}{\partial z}=0, z=0
$$

$\widehat{\tau}_{13}+i \alpha \widehat{T}=0, \widehat{\tau}_{23}+i \beta \widehat{T}=0, \widehat{w}=0, \frac{\partial \widehat{T}}{\partial z}=0, z=1$.

Substituting (2.13) into governing Eqs. (2.3)-(2.6), the linearized perturbation equations can be derived as follows:

$$
i \alpha \widehat{u}+i \beta \widehat{v}+D \widehat{w}=0,
$$

$$
\begin{gathered}
R\left[\beta\left(\widehat{w} D U_{0}+U_{0} i \alpha \widehat{u}\right)-\alpha\left(U_{0} i \alpha \widehat{v}\right)\right]-\beta\left(i \alpha \widehat{\tau}_{11}+i \beta \widehat{\tau}_{12}+D \widehat{\tau}_{13}\right)+\alpha\left(i \alpha \widehat{\tau}_{12}+i \beta \widehat{\tau}_{22}+D \widehat{\tau}_{23}\right)=-\sigma R(\beta \widehat{u}-\alpha \widehat{v}), \\
R \alpha\left(D \widehat{w} \cdot D U_{0}+\widehat{w} D^{2} U_{0}+D U_{0} \cdot i \alpha \widehat{u}+U_{0} i \alpha D \widehat{u}\right)+R \beta\left(D U_{0} \cdot i \alpha \widehat{v}+U_{0} i \alpha D \widehat{v}\right)-R i k^{2}\left(U_{0} i \alpha \widehat{w}\right) \\
-\left(i \alpha{ }^{2} D \widehat{\tau}_{11}+2 i \alpha \beta D \widehat{\tau}_{12}+\alpha D^{2} \widehat{\tau}_{13}+i \beta{ }^{2} D \widehat{\tau}_{22}+\beta D^{2} \widehat{\tau}_{23}\right)+i k^{2}\left(i \alpha \widehat{\tau}_{13}+i \beta \widehat{\tau}_{23}+D \widehat{\tau}_{33}+B o \cdot \widehat{T}\right) \\
=-\sigma R\left(\alpha D \widehat{u}+\beta D \widehat{v}-i k^{2} \widehat{w}\right), \\
M a\left(\widehat{u} \frac{\partial T_{0}}{\partial x}+\widehat{w} \frac{\partial T_{0}}{\partial z}+U_{0} i \alpha \widehat{T}\right)+\left(\alpha 2+\beta^{2}\right) \widehat{T}-D^{2} \widehat{T}=-\sigma M a \widehat{T} \\
\widehat{\tau}_{11}-\mu 2 i \alpha \widehat{u}=0, \widehat{\tau}_{12}-\mu(i \alpha \widehat{v}+i \beta \widehat{u})=0, \widehat{\tau}_{13}-\mu_{t}(D \widehat{u}+i \alpha \widehat{w})=0 \\
\widehat{\tau}_{22}-\mu(2 i \beta \widehat{v})=0, \widehat{\tau}_{23}-\mu(i \beta \widehat{w}+D \widehat{v})=0, \widehat{\tau}_{33}-\mu(2 D \widehat{w})=0 .
\end{gathered}
$$


TABLE I. The critical parameters for Newtonian fluid.

\begin{tabular}{lcccc}
\hline \hline $\operatorname{Pr}=0.1$ & $M a$ & $k$ & $\psi(\mathrm{deg})$ & $c$ \\
\hline Linear flow & 12.0 & 0.91 & 83.4 & 0.046 \\
Return flow & 22.4 & 0.65 & 71.2 & 0.070 \\
\hline \hline
\end{tabular}

Then, the Chebyshev collocation method is used to solve the eigenvalue problem with the form of $\mathbf{W g}=\sigma \mathbf{Z g}$, where $\mathbf{W}$ and $\mathbf{Z}$ are two matrices and $\mathbf{g}$ is the eigenvector. ${ }^{31}$ The eigenvalues are obtained by using the QZ algorithm. We use more than 80 Chebyshev nodes to ensure the accuracy in the following. In order to validate our code, we solve the same problem of Newtonian fluid by setting $n=1$ and compare the results with those in the previous work. The critical parameters of Newton fluid are listed in Table I, which agree with the results in Ref. 26 . Here, $\psi=180^{\circ}-\phi$ and $c=\left|\sigma_{i}\right| / k$ is the wave speed.

\section{NUMERICAL RESULTS}

We compute the Marangoni number $M a_{N}$ of the neutral modes $\left(\sigma_{r}=0\right)$. Then the critical Marangoni number $M a_{c}$ is obtained as follows:

$$
M a_{c}=\min _{\alpha, \beta} M a_{N}(\operatorname{Pr}, \lambda, n, B o) .
$$

\section{A. Linear flow}

For linear flow, the viscosity $\mu$ and tangent viscosity $\mu_{t}$ are homogeneous in $z$. It is more convenient to use the viscosity in the flow instead of $\hat{\mu}_{0}$ for the definition of the Marangoni number and Prandtl number. Thus we use the following numbers for linear flow:

$$
M a_{e c}=M a_{c} / \mu, P r_{e}=\operatorname{Pr} \cdot \mu .
$$

We plot the variation of $M a_{e c}$ with $\operatorname{Pr}_{e}$ for linear flow at various values of $n, \lambda$ in Fig. 4 . The preferred modes are oblique wave $\left(\phi \neq 0^{\circ}, 90^{\circ}\right)$, streamwise wave $\left(\phi=0^{\circ}\right)$, and spanwise stationary mode $\left(\phi=90^{\circ}, \sigma_{i}=0\right)$ for small, moderate, and large $\operatorname{Pr}_{e}$, respectively. Comparing with Newtonian fluid $(\lambda$ $=0$ or $n=1)$, the shear-thinning effect leads to a decreasing

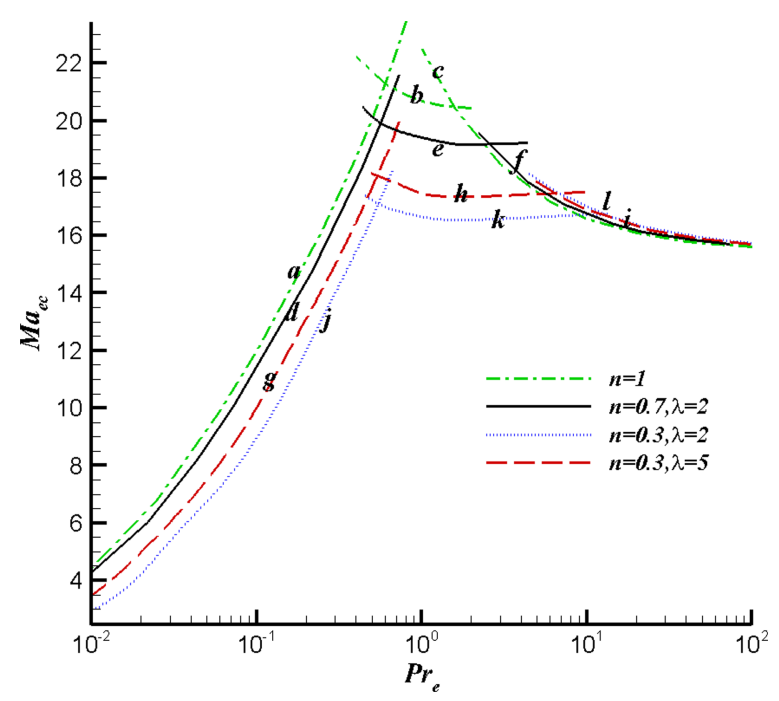

FIG. 4. The variation of $M a_{e c}$ with $\mathrm{Pr}_{e}$ for linear flow. The curves correspond to (1) oblique wave: (a), (d), (g), and (j); (2) streamwise wave: (b), (e), (h), and (k); (3) spanwise stationary mode: (c), (f), (i), and (1).

of $M a_{e c}$ at small and moderate $\operatorname{Pr}_{e}$ while there is a little increasing of $M a_{e c}$ at large $P r_{e}$. This leads to the variation of bifurcation points: the preferred mode changes from oblique wave to streamwise wave at almost the same $P r_{e}$, while the bifurcation points from streamwise wave to spanwise stationary mode is changed obviously by the effect of shearthinning.

When $n=0.3$, we can find that $\left.M a_{e c}\right|_{\lambda=2}<\left.M a_{e c}\right|_{\lambda=5}$ $<\left.M a_{e c}\right|_{\lambda=0}$ at small and moderate $P r_{e}$, which indicates that the effect of $\lambda$ for the flow stability is not monotonous. Similar phenomenon can also be found for return flow in Sec. III B and the case of channel flow. ${ }^{23}$ The reason is that when $\lambda$ is larger enough, the Carreau model reduces to the power-law model: $\mu=(\lambda \dot{\gamma})^{n-1}$. Then, the shearing thinning effect only depends on $n$, while the value of $\lambda$ can be normalized in the nondimensionalization. Therefore, the effect of shear-thinning on flow stability is more obvious at moderate $\lambda$.

The wave number and propagation angle corresponding to the modes in Fig. 4 are displayed in Fig. 5. The changes of wave number caused by shear-thinning are similar to those of $M a_{e c}$. The wave propagation angle at $n=0.7$ is larger than

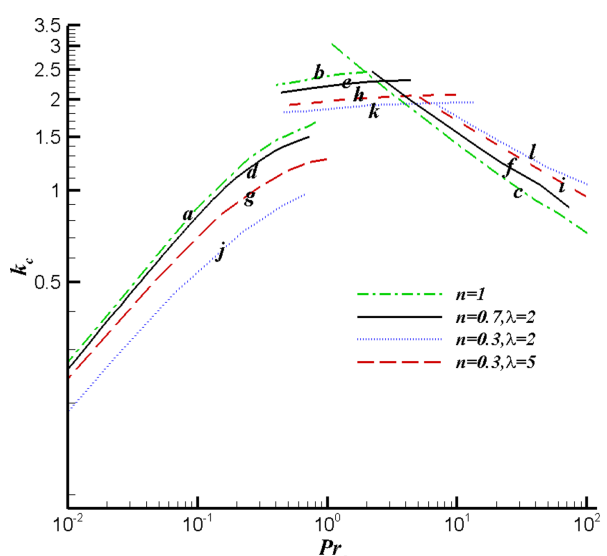

(a)

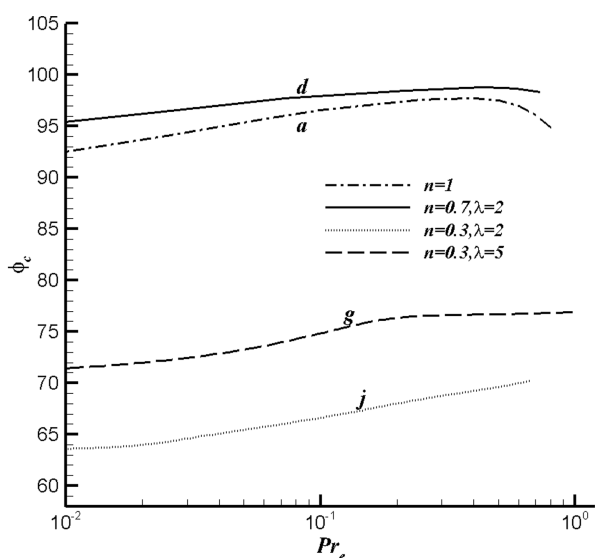

(b)
FIG. 5. The (a) wave number and (b) wave propagation angle corresponding to the modes in Fig. 4. 
that of Newtonian fluid. In contrast, $\phi_{c}$ decreases at $n=0.3$ and the preferred mode changes from upstream to downstream. This difference is associated with the heat transfer. For the oblique wave of Newtonian fluid, the wave is close to spanwise. The key to the mechanism is the horizontal convection $u \frac{\partial T_{0}}{\partial x}$, which promotes an upstream wave. As $n<1$, it can be seen from (2.16) and (2.17) that $\mu_{t} \leq \mu$ (the equality holds where the shear rate of basic flow $\dot{\gamma}_{0}=0$ ). Then, the amplitude of $\widehat{u}$ increases, which leads to an increase in $u \frac{\partial T_{0}}{\partial x}$. This is the reason why the propagation angle of the upstream wave is closer to the streamwise direction for $n=0.7$. However, for $n=0.3$, the importance of heat convection induced by the basic flow $U_{0} \frac{\partial T}{\partial x}$ rises as $\left|\phi_{c}-90^{\circ}\right|$ is no longer small. This term promotes a downstream wave, which is opposite to $u \frac{\partial T_{0}}{\partial x}$.

\section{B. Return flow}

For return flow, the shear rate is not homogeneous. So there is a viscosity stratification for shear-thinning fluid. Comparing the perturbation of Carreau fluid with that of Newtonian fluid, the amplitude of horizontal velocity is an order of magnitude larger than that of vertical velocity for both of them. However, the kinetic energy of perturbation for the former is more concentrated near the surface, which can be seen in Fig. 6. This concentration attributes to the effect of shearthinning. Suppose a perturbation mode in Newtonian fluid is added in the Carreau fluid, then, as the shear-thinning effect makes the viscosity near the surface much smaller, there is less viscous dissipation for the perturbation near the surface. Thus, the kinetic energy of perturbation increases obviously in this region. The viscosity also decreases near the wall. Therefore, the energy growth by the shear-thinning effect is obvious near both the surface and the wall (see Fig. 6). However, the solid boundary suppresses the growth of perturbation energy, and the kinetic energy near the surface is much larger than that near the wall. Finally, the perturbation energy for Carreau fluid is more concentrated near the surface.

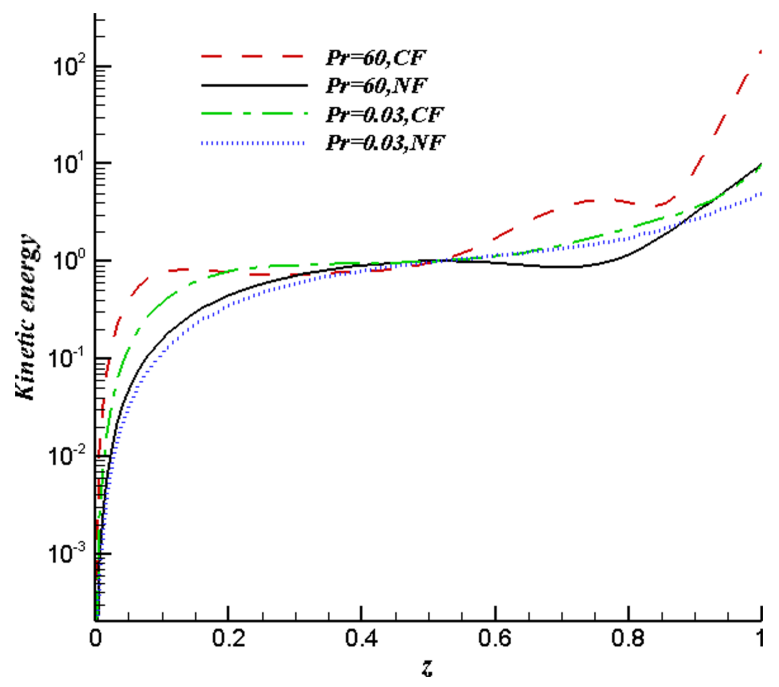

FIG. 6. The distributions of the kinetic energy of preferred mode for return flow. NF and CF stand for Newtonian fluid $(n=1)$ and Carreau fluid at $n$ $=0.3, \lambda=5$, respectively. The kinetic energy is normalized by the value at $z$ $=0.5$.

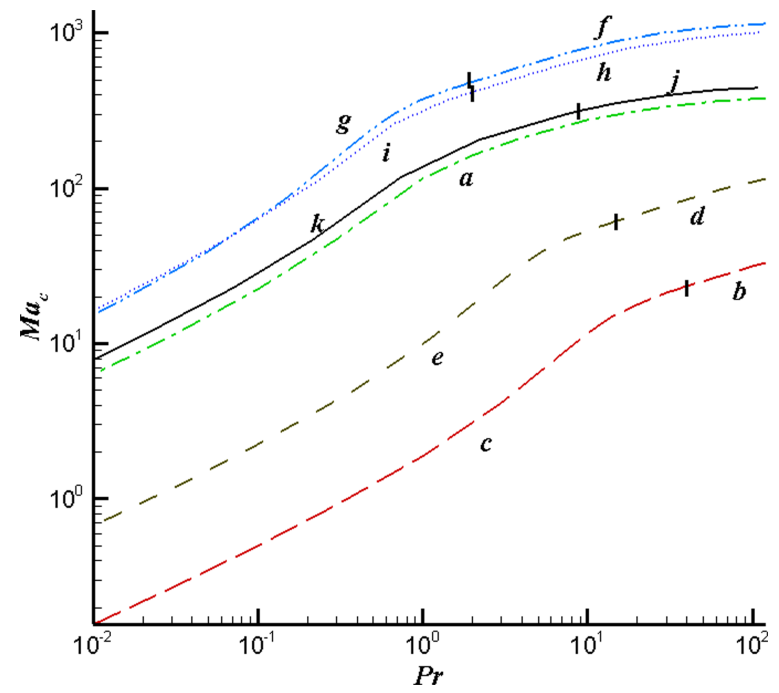

FIG. 7. The variations of critical Marangoni number with Prandtl number for return flow. The curves correspond to (1) $M a_{c}$ vs $\operatorname{Pr}$ at $n=1$ (Newtonian fluid): (a) oblique wave; (2) $M a_{c}$ vs $\operatorname{Pr}$ at $n=0.3, \lambda=5$ : (b) streamwise wave and (c) oblique wave; (3) $\overline{M a_{c}}$ vs $\overline{P r}$ at $n=0.3, \lambda=5$ : (d) streamwise wave and (e) oblique wave; (4) $M a_{w c}$ vs $P r_{w}$ at $n=0.3, \lambda=5$ : (f) streamwise wave and (g) oblique wave; (5) $M a_{w c}$ vs $P r_{w}$ at $n=0.3, \lambda=2$ : (h) streamwise wave and (i) oblique wave; (6) $M a_{w c}$ vs $P r_{w}$ at $n=0.7, \lambda=2$ : (j) streamwise wave and (k) oblique wave.

The variations of critical Marangoni number with Prandtl number for return flow are plotted in Fig. 7. Here, $M a_{c}, \overline{M a_{c}}$, and $M a_{w c}$ correspond to the critical Marangoni number based on the viscosity $\hat{\mu}_{0}$, the average viscosity across the layer, and the viscosity on the surface, respectively. The definitions of $P r$, $\overline{P r}$, and $P r_{w}$ are similar. It can be seen that $M a_{c}$ and $\overline{M a_{c}}$ of Carreau fluid [curve (b)-(e) in Fig. 7] are smaller than $M a_{c}$ of Newtonian fluid [curve (a) in Fig. 7]. It seems that the shearthinning effect makes the flow more unstable. However, the kinetic energy of perturbation concentrates near the surface for return flow. If we use the viscosity on the surface for the definition of Marangoni number and Prandtl number, it can be found that $M a_{w c}$ of Carreau fluid [curve (f)-(k) in Fig. 7] are larger than $M a_{c}$ of Newtonian fluid [curve (a) in Fig. 7]. Thus, the shear-thinning effect stabilizes the flow when the viscosity on the surface is applied. This agrees with the result of Ref. 23 for channel flow. However, as $P r_{w}$ depends on the shear-rate, we will still use $P r$ in the following, which only depends on the property of the fluid.

In Fig. 8, we plot the wave number and the wave propagation angle corresponding to the preferred mode of Carreau fluid in Fig. 7. The wave number for oblique wave decreases with the extent of shear-thinning, while for streamwise wave, the variation is not monotonous. There are two main changes of the propagation angle in Carreau fluid: for large $P r$, the preferred mode becomes the upstream streamwise wave, which has not been found in Newtonian fluid (Fig. 7); while for small $\mathrm{Pr}$, the propagation angle increases [see Fig. 8(b)]. For curve (c) in Fig. 8(b), the propagation angle of the oblique wave can reach $180^{\circ}$ for a critical value of $P r$. When $\operatorname{Pr}$ exceeds this value, the preferred mode changes from the oblique wave to the upstream streamwise wave [curve (b) in Fig. 7], whose propagation angle is $\phi_{c}=180^{\circ}$. The cases for curve (i) and (k) in Fig. 8(b) are similar. 


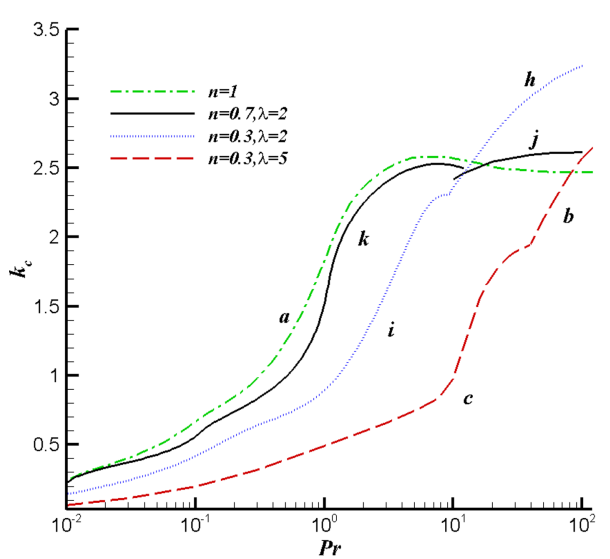

(a)

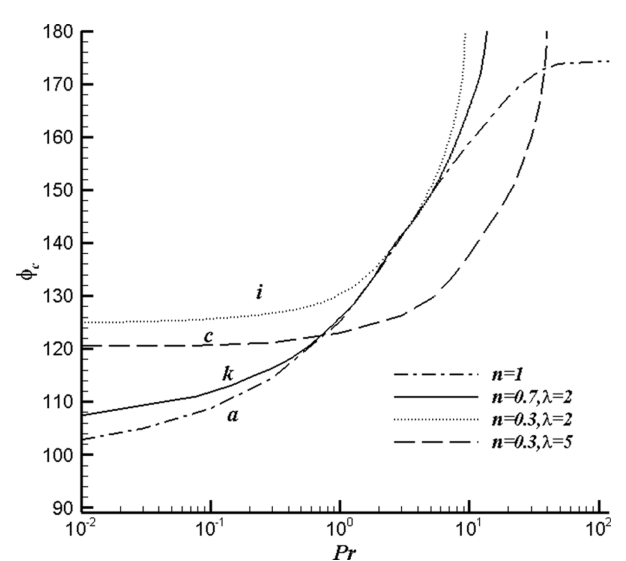

(b)
FIG. 8. The (a) wave number and (b) wave propagation angle corresponding to the mode in Fig. 7.
Then we pay attention to the perturbation flow field of return flow. The streamlines and isothermals of preferred mode at $\operatorname{Pr}=100, \operatorname{Pr}=0.1$, and $P r=10$ are plotted in Figs. 9-11, respectively. Although the shear-thinning effect changes the critical parameters for small and large Prandtl numbers obviously, the perturbation flow fields of Carreau fluid at $\operatorname{Pr}=100$ and $P r=0.1$ are similar to those of Newtonian fluid. ${ }^{32}$ The former (Fig. 9) has interior hot spots, which absorbs energy from the vertical convection and heats the interface by conduction. Then the temperature of the surface upon the hot spot increases and the wave propagates upstream. In contrast, the latter (Fig. 10) has vertical isothermals and the distribution of surface temperature is the same as that in the layer. The key to the instability mechanism is the inertially driven streamwise flow. ${ }^{32}$

A new instability mechanism that differs from Newtonian fluid has been found for $\operatorname{Pr}=10$, where the amplitude of temperature is at the bottom (see Fig. 11). The hot spot at the bottom is heated by the horizontal convection, which includes $U_{0} \frac{\partial T}{\partial x}$ and $u \frac{\partial T_{0}}{\partial x}$. These two terms have the same

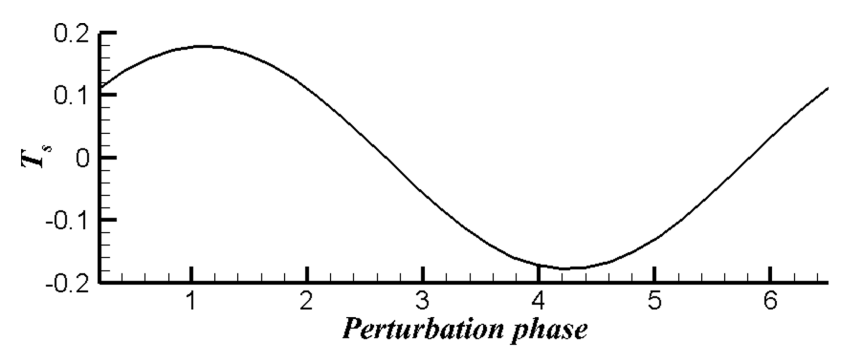

(a)

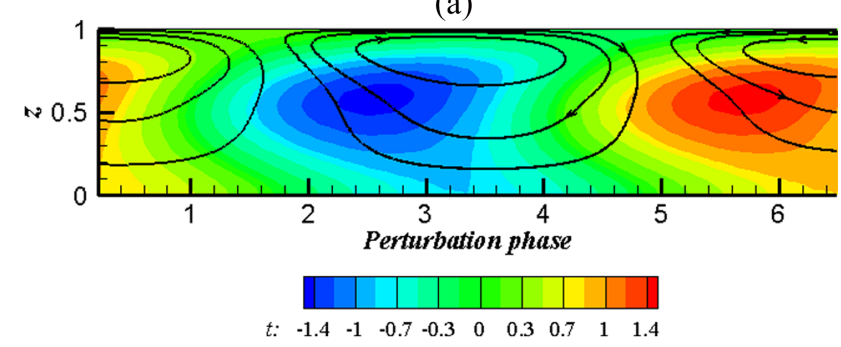

(b)

FIG. 9. The perturbation flow field of the streamwise wave for Carreau fluid at $P r=100, M a=31.47, n=0.3, \lambda=5, B o=0$ : (a) the distribution of surface temperature; (b) the streamlines and isothermals. order and sign. They are related to the velocity and temperature gradient of the basic flow, respectively. In contrast, the hot spot on the surface is induced by the heat conduction from the interior. Thus, the key to the instability mechanism is the hot spot at the bottom, which absorbs energy from the horizontal convection in the lower region of layer and drives the perturbation wave by conductively heating the interface.

The temperature distribution in Fig. 11 is caused by the combination of the convection in the lower region and the conduction near the surface. The hot spot at the bottom is heated by the horizontal convection in the lower region. This indicates that the convection is dominant there. In contrast, the surface above the hot spot is cooled by the convection as the signs of $U_{0} \frac{\partial T}{\partial x}$ and $u \frac{\partial T_{0}}{\partial x}$ are both opposite to those near the wall. If the convection is still dominant near the surface, this leads to a cold spot on the surface, which is just opposite to the case in Fig. 11. Thus, the hot spot on the surface can only absorbs energy by the heat conduction from the interior.

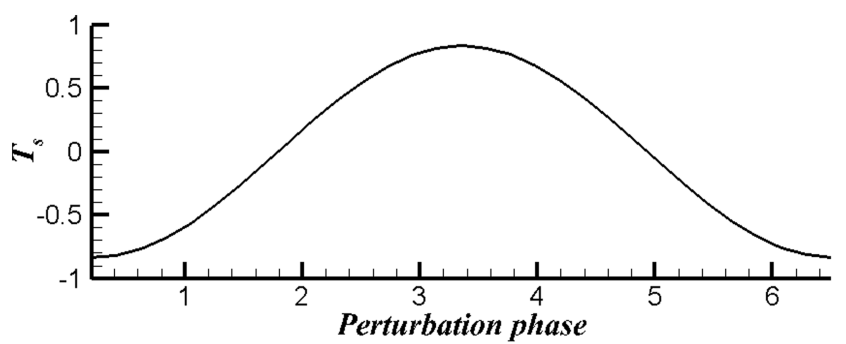

(a)

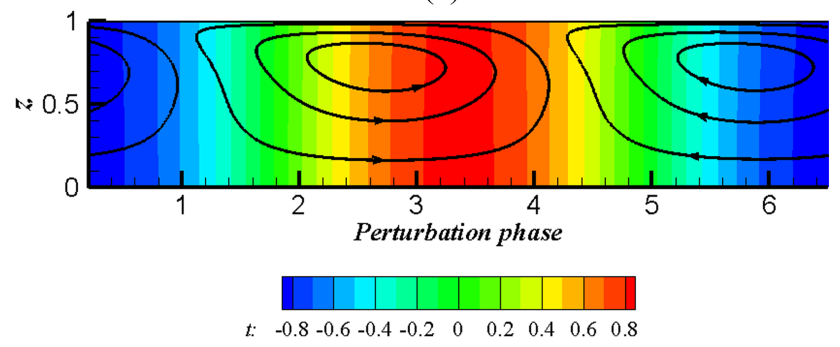

(b)

FIG. 10. The perturbation flow field of the oblique wave for Carreau fluid at $P r=0.1, M a=0.499, n=0.3, \lambda=5, B o=0$ : (a) the distribution of surface temperature; (b) the streamlines and isothermals. 


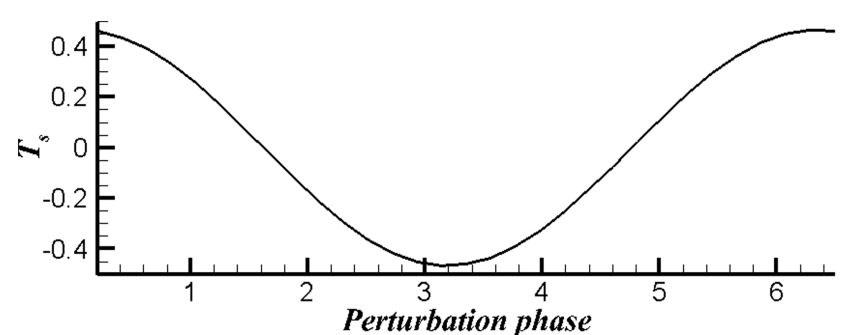

(a)

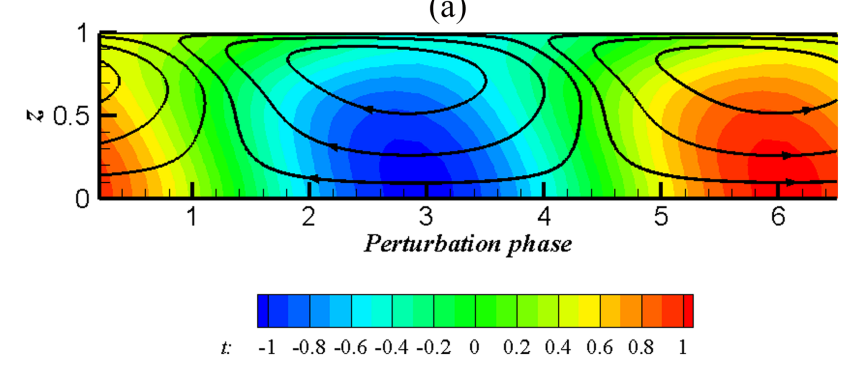

(b)

FIG. 11. The perturbation flow field of the oblique wave for Carreau fluid at $\operatorname{Pr}=10, M a=11.55, n=0.3, \lambda=5, B o=0$ : (a) the distribution of surface temperature; (b) the streamlines and isothermals.

The combination of convection and conduction is associated with the stratification of the Prandtl number $\operatorname{Pr}_{e}(z)$ $=\operatorname{Pr} \cdot \mu$, which is defined in (3.1) and stands for the distribution of local Prandtl number in the flow. In Fig. 11, $\operatorname{Pr}_{e}$ $\approx 0.48$ on the surface, so the conduction is more important for the heat transfer. However, $\operatorname{Pr}_{e} \approx 9$ in the region $z$ $=0.3$, where the heat convection is dominant. The mechanism in Fig. 11 is obvious when $\operatorname{Pr} \in(1,20)$ at $n=0.3, \lambda$ $=5$. When $\operatorname{Pr}$ is outside this range, it has $\max \operatorname{Pr}_{e}(z)<1$ or $\min _{z} \operatorname{Pr}_{e}(z)>1$ in the flow, then the combination of the convection in the lower region and the conduction near the surface will degenerate.

The stratification of local Prandtl number is made by the viscosity stratification in shear-thinning fluid. As the shearthinning effect makes viscosity stratification in return flow, $\operatorname{Pr}_{e}$ is also stratified, which has a great impact on the heat transfer. Finally, we can observe that the stratification of $\mathrm{Pr}_{e}$ in shearthinning fluid is crucial for the temperature distribution in Fig. 11. This is the reason why it does not appear in previous studies for other fluids.

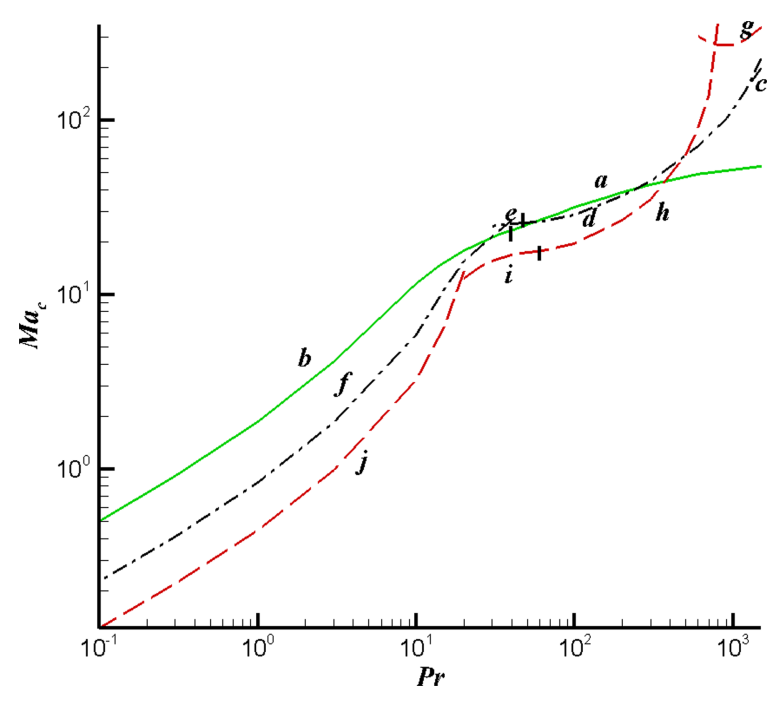

FIG. 12. The variation of $M a_{c}$ with $\operatorname{Pr}$ for return flow at $n=0.3, \lambda=5$ in the presence of gravity. The curves corresponds to (1) $B o=0$ : (a) streamwise wave and (b) oblique wave; (2) $B o=3$ : (c) streamwise wave with $k_{c}>2.9$, (d) streamwise wave with $k_{c}<1.1$, (e) oblique wave with $\phi_{c}<60^{\circ}$, and (f) oblique wave with $\phi_{c}>100^{\circ}$; (3) $B o=6$ : (g) streamwise wave with $k_{c}>2$, (h) streamwise wave with $k_{c}<1.1$, (i) oblique wave with $\phi_{c}<102^{\circ}$, and (j) oblique wave with $\phi_{c}>117^{\circ}$.

\section{Effect of gravity}

We will study the effect of gravity in this section. The variation of $M a_{c}$ with $\mathrm{Pr}$ for return flow is displayed in Fig. 12 at different gravity levels. $M a_{c}$ increases with $B o$ at large $P r$, while the situation is opposite at small $\mathrm{Pr}$. The wave number and the wave propagation angle corresponding to the mode in Fig. 12 are displayed in Fig. 13.

It is observed that more kinds of preferred modes are excited by gravity. There are two kinds of streamwise wave and oblique wave for both $B o=3$ and $B o=6$. For oblique waves in Fig. 13(b), the first kind is downstream when $\mathrm{Pr}$ $>30$, whose propagation angle decreases with $\mathrm{Pr}$. The second is upstream. The variation of its propagation angle with $\operatorname{Pr}$ is not monotonous. The wave number and propagation angle of oblique wave are not sensitive to the change of $\mathrm{Bo}$ when $\mathrm{Pr}$ $<1$, although the distributions of basic flow and viscosity have changed obviously.

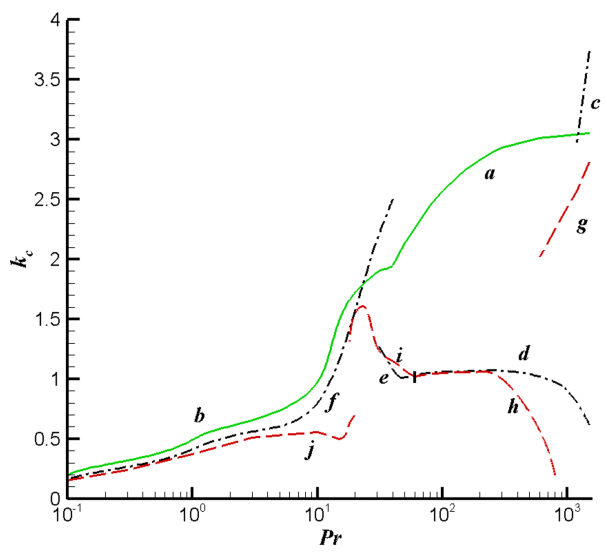

(a)

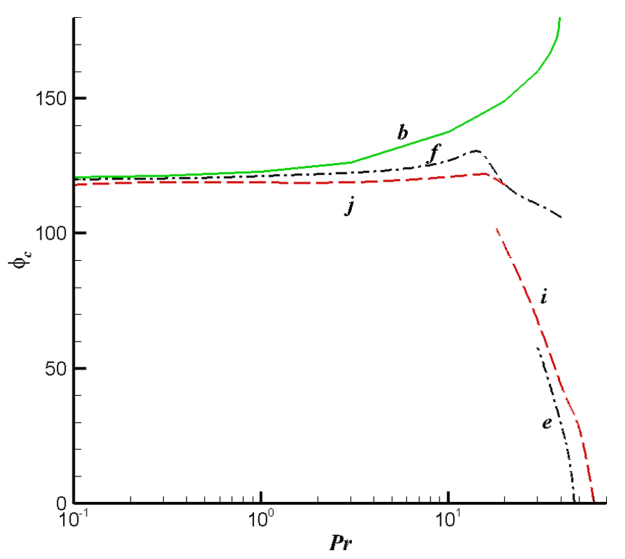

(b)
FIG. 13. The (a) wave number and (b) wave propagation angle corresponding to the mode in Fig. 12. 


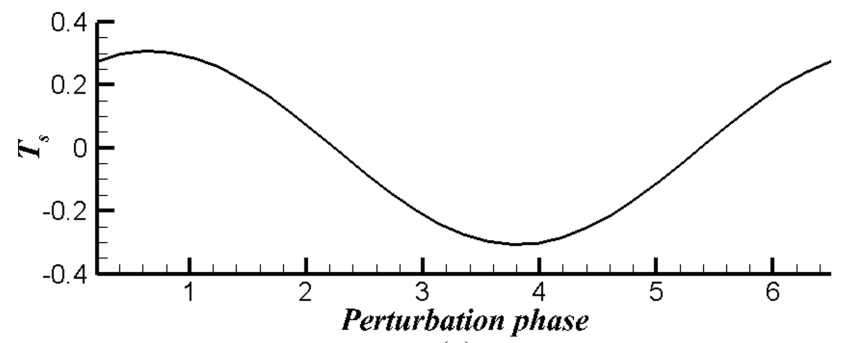

(a)

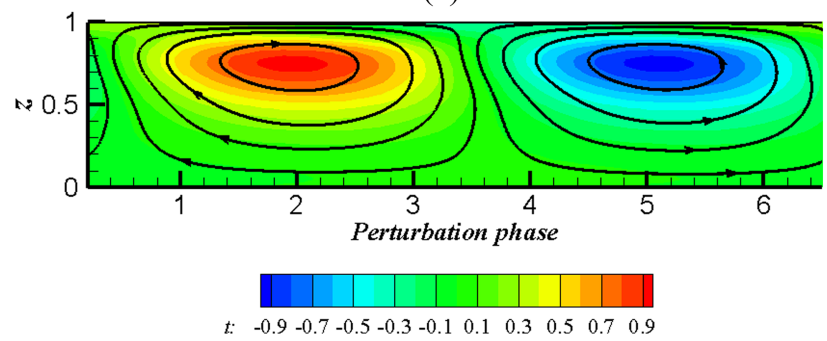

(b)

FIG. 14. The perturbation flow field of the streamwise wave for Carreau fluid at $P r=900, M a=101.9, n=0.3, \lambda=5, B o=3$ : (a) the distribution of surface temperature; (b) the streamlines and isothermals.

The variations of wave number with $\mathrm{Pr}$ are opposite for two kinds of streamwise waves [see Fig. 13(a)]. However, both of them propagate downstream, which differs from the case without gravity. The perturbation flow field is plotted in Fig. 14 for return flow at $B o=3$. It shows that the streamlines near the hot spot are clockwise and almost coincide with the isothermals. In contrast, the corresponding streamlines are counterclockwise and staggered with the isothermals in the case without gravity (see Fig. 9).

The reason for the change of propagation direction can be explained as follows. In Fig. 14, the surface upon the hot spot is heated by horizontal convection $U_{0} \frac{\partial T}{\partial x}, u \frac{\partial T_{0}}{\partial x}$, and the vertical conduction $\frac{1}{M a} \frac{\partial^{2} T}{\partial z^{2}}$. When $B o$ increases, the heat convection induced by basic flow $U_{0} \frac{\partial T}{\partial x}$ on the surface becomes more important as the gravity increases $U_{0}$ on the surface significantly [see Fig. 2(a)]. This term heats the downstream point of hot spot on the surface and promotes a downstream wave.

\section{Energy analysis}

Then we study the energy mechanism in this section. The rate of change for perturbation energy can be written as ${ }^{17,33}$

$$
\begin{aligned}
\frac{\partial E_{k i n}}{\partial t}= & -\frac{1}{2 R} \int(\boldsymbol{\tau}: \dot{\boldsymbol{\gamma}}) \mathrm{d}^{3} r+\frac{1}{R} \int \mathbf{u} \cdot \boldsymbol{\tau} \cdot \mathbf{n} \mathrm{d}^{2} r \\
& -\int \mathbf{u} \cdot\left((\mathbf{u} \cdot \nabla) \mathbf{u}_{0}\right) \mathrm{d}^{3} r+\int\left(\frac{B o}{R} T \mathbf{e}_{3} \cdot \mathbf{u}\right) \mathrm{d}^{3} r \\
= & -N+M+I+G,
\end{aligned}
$$

where $N$ is the work done by the perturbation stress, $M$ is the work done by Marangoni forces on the surface, $I$ is the interaction between the perturbation flow and the basic flow, $G$ is the work done by gravity.

It can be indicated from (2.15) that $N>0$ for Carreau fluid. Thus, this term stands for the viscous dissipation. The perturbation energy can come only from the last three terms in (3.3). It should be noted that there is a big difference between the energy mechanism of thermocapillary liquid layer and that of channel flow. For the former, $M$ is a important energy source for perturbation, ${ }^{34}$ while for the latter (solid boundaries), the perturbation energy can come only from $I$ in the absence of gravity.

We define $S$ as the work done by the perturbation stress $\tau_{13}$,

$$
S=\frac{1}{2 R} \int\left(\tau_{13} \cdot \dot{\gamma}_{13}\right) \mathrm{d}^{3} r .
$$

The effect of shear-thinning and gravity for energy mechanism can be seen from $R i, R s, R g$, which are the relative sizes of $I$, $S, G$ to $N$,

$$
R i=I / N, R s=S / N, R g=G / N
$$

In Table II, the terms in (3.5) of preferred modes are listed for return flow without gravity. It can be found that the shearthinning makes $R s$ increasing obviously for most of $P r$. The change of $R s$ can be explained as follows. As $\mu_{t}$ is smaller than $\mu$, the amplitude of $\dot{\gamma}_{13}$ increases in Carreau fluid under the same shear force, which leads to the growth of viscous dissipation made by $\dot{\gamma}_{13}$.

The effect of shear-thinning also leads to the growth of $R i$ at large $P r$. However, the change of $R i$ at small $P r$ is not apparent. It is known from previous studies that $R i$ becomes important at small $P r$, while it is negligible at large $P r \cdot{ }^{34} \mathrm{In}$ shear-thinning fluid, as the viscosity is smaller than $\hat{\mu}_{0}$ in most of the flow region, the effect value of $\operatorname{Pr}$ decreases, which leads to a rising of $R i$. However, the increase of $R i$ stops when $P r$ is small.

The terms in (3.5) of preferred modes are plotted in Fig. 15 for return flow at $B o=3$. $R g$ is negligible at large $P r$. Therefore, the effect of gravity is only changing the basic flow in this region. However, $R g$ can reach $12.5 \%$ at small $P r$, which is important for the energy mechanism. When $\mathrm{Pr}>50, R i$ increases with $P r$ and finally changes from negative to positive, while it keeps positive when $\operatorname{Pr}<30$.

In Fig. 15, the variations at large and small $P r$ are gentle. In contrast, there are severe changes when $50 \geq \operatorname{Pr} \geq 10$, and the preferred mode also changes two times in this region. This may due to the mechanism of heat transfer. The heat convection is more significant than the heat conduction for the temperature field at large $P r$ while the opposite appears at small $P r$. The importance of convection and conduction are comparable at moderate $P r$, which makes the instability mechanism more

\begin{tabular}{|c|c|c|c|c|}
\hline \multirow[b]{2}{*}{$P r$} & \multicolumn{2}{|c|}{$n=0.3, \lambda=5$} & \multicolumn{2}{|c|}{$n=1$} \\
\hline & $R i$ & $R s$ & $R i$ & $R s$ \\
\hline 300 & 0.0055 & 0.6966 & 0.0000 & 0.5769 \\
\hline 100 & 0.0252 & 0.7302 & 0.0004 & 0.5757 \\
\hline 30 & 0.0948 & 0.7396 & 0.0035 & 0.5683 \\
\hline 3 & 0.1563 & 0.4217 & 0.1024 & 0.4006 \\
\hline 0.3 & 0.1600 & 0.3974 & 0.1626 & 0.2500 \\
\hline 0.03 & 0.1619 & 0.4035 & 0.1514 & 0.1862 \\
\hline
\end{tabular}
complicated and sensitive to $P r$.

TABLE II. The terms in (3.5) of preferred modes for return flow without gravity. Comparisons are made with Carreau fluid at $n=0.3, \lambda=5$ and Newtonian fluid $(n=1)$. 


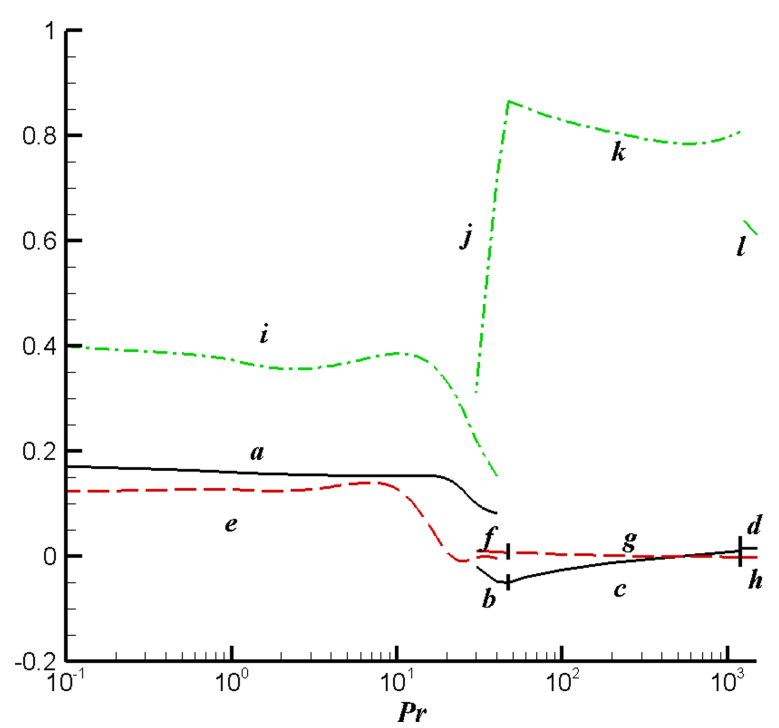

FIG. 15. The terms in (3.5) of preferred modes for return flow at $n=0.3, \lambda$ $=5, B o=3$. The curves correspond to (1) Ri: (a)-(d); (2) Rg: (e)-(h); (3) Rs: (i)-(1).

\section{DISCUSSION}

We will discuss the influence of shear-thinning effect on the instability mechanism and make comparison with channel flows in this section.

\section{A. Linear flow}

In the absence of viscosity stratification, the linear flow only yields an anisotropic disturbance stress tensor, which is the same as the property of plane Couette flow. However, the former is driven by the thermocapillary force on the surface while the latter is a shear flow between two plates. Thus, their stabilities are also different. Liu and $\mathrm{Liu}^{24}$ have shown that the effect of shear-thinning is destabilizing for the plane Couette flow. In contrast, the situation for thermocapillary flow depends on the Prandtl number: the flow is destabilized by shear-thinning at small and moderate $\mathrm{Pr}_{e}$ while the flow stability is increased slightly at large $P r_{e}$.

These can be explained by the property of preferred mode. Previous studies ${ }^{26,32}$ have shown that the horizontal and vertical convections are crucial for the instability mechanism of streamwise (moderate $P r_{e}$ ) and oblique waves (small $P r_{e}$ ), respectively. Both of these two convections are enhanced by the shear-thinning effect, as the amplitude of perturbation velocities in $x$ and $z$ directions are increased [see (2.15), the viscosity corresponding to $\dot{\gamma}_{13}$ becomes smaller in shear-thinning fluid]. Thus, the flow becomes more unstable. Meanwhile, the decreasing of the Marangoni number will increase the importance of heat conduction [see (2.6)], which dissipates the temperature perturbation. It can be balanced by the decreasing of wave number. So the wave number of the preferred mode decreases in Fig. 5(a). This explanation also holds in Figs. 8(a) and 13(a), where the wave number of the oblique wave is also decreased with the decreasing of critical Marangoni number by the shear-thinning effect.

For spanwise stationary mode (large $\left.P r_{e}\right), \mu_{t}$ only affects $\hat{u}$. Comparing with Newtonian fluid, the viscous resistance in streamwise direction is reduced at the same Marangoni number. As a result, the amplitude of $\hat{u}$ increases. The computation shows that the streamwise velocity $u<0$ when underneath the hot spot on the surface, which produces a convective cooling. So the effect of $\mu_{t}$ makes the flow more stable. However, the increasing of critical Marangoni number is very slight. The reason is that the vertical convection is dominant for the temperature field, and the change in horizontal convection does not have a serious impact. The increasing of convective cooling by the shear-thinning effect can be balanced by the increase in vertical convection. The latter can be achieved for a higher wave number, which leads to an increase in the vertical velocity [see (2.20)]. So we can find that the wave number of spanwise stationary mode is increased in Fig. 5(a).

\section{B. Return flow}

The shear-thinning effect makes the viscosity stratified in return flow, which is similar to the case of plane Poiseuille flow. However, the former has a free boundary on the surface while the latter has two solid boundaries. The perturbation energy of them mainly comes from the thermocapillary force and basic flow, respectively. Therefore, the instability of the return flow is closely related to the surface and temperature field, which differs from the channel flow.

Nouar et $a l .{ }^{23}$ have stated that the shear-thinning effect stabilizes the plane Poiseuille flow when an appropriate viscosity (the wall tangent viscosity and the effective viscosity at the wall) is used in the definition of the Reynolds number. In the present work, it is found that the flow becomes more stable in shear-thinning fluid when the viscosity on the surface is used, which agrees with the result of Nouar et $a .^{23}$ It can be explained from the energy mechanism. For the liquid layer, $I$ is not the main energy source, and there is an approximate balance between $N$ and $M$ for the neutral mode,

$$
\frac{1}{2 R} \int(\boldsymbol{\tau}: \dot{\gamma}) \mathrm{d}^{3} r \approx \frac{1}{R} \int \mathbf{u} \cdot \boldsymbol{\tau} \cdot \mathbf{n} \mathrm{d}^{2} r
$$

Due to the viscosity stratification, the viscosity in the interior is larger than that on the surface. Thus, the left side of (4.1) increases in shear-thinning fluid, and the flow becomes more stable.

For Newtonian fluid, the preferred mode is oblique wave at all Prandtl numbers. ${ }^{26}$ However, for Carreau fluid, the preferred mode becomes the upstream streamwise wave at large Prandtl number, and the propagation angle increases at small Prandtl number. The shear-thinning effect increases the horizontal convection $u \frac{\partial T_{0}}{\partial x}$, which promotes an upstream wave. As a result, the propagation direction becomes closer to the negative $x$-axis.

The viscosity stratification also excites a new instability mechanism of return flow. Due to the viscosity stratification, the relative size of viscosity to thermal diffusivity varies significantly in the vertical direction at moderate Prandtl number, which is crucial for the heat transfer. Thus, one would expect that the temperature field of Newtonian fluid and Carreau fluid may be very different in this region. This is confirmed in Fig. 11 where the hot spots appear at the bottom for Carreau 
fluid. The mechanism is a combination of horizontal convection in the lower region of the layer and heat conduction near the surface. In contrast, the hot spots are in the middle of the layer at moderate Prandtl number for Newtonian fluid.

\section{Effect of gravity}

The flow stability can be affected by gravity in two ways. The first is the change in basic flow. We can observe in Fig. 2 that the extent of shear thinning is enhanced by gravity. However, the values of $\mu$ and $\mu_{t}$ at $z=1$ keep the same at different gravity level (see Fig. 3). The second is the work done by gravity for the perturbation energy.

More kinds of preferred modes are excited by gravity. When $\operatorname{Pr}$ is very large, $M a_{c}$ increases with $B o$ significantly, which leads to the remarkable growth for the gravity and thermocapillary forces together. These two forces are coupled and excite new modes. For moderate Prandtl number, the change for the relative size of viscosity to thermal diffusivity in the vertical direction is enhanced by gravity, which has a great impact on the heat transfer. So the opportunity for the change of preferred mode also increases.

For large $\operatorname{Pr}$, the work done by gravity is negligible while the perturbation flow field changes significantly. In the presence of gravity, the preferred mode changes from upstream to downstream while the streamlines near the hot spot changes from counterclockwise to clockwise, which are both related to the increasing of $U_{0}$ on the surface. In contrast, for small $\mathrm{Pr}$, the gravity becomes an important perturbation energy source while the change in the perturbation field is not apparent. The reason can be explained as follows. The expression of $G$ in (3.3) indicates that the work done by gravity is associated with perturbation velocity in the vertical direction. For large $\mathrm{Pr}$, most of the perturbation energy is concentrated in a thin layer near the surface, where the vertical velocity of perturbation is very small. So $G$ is negligible. Instead, the concentration at small $\mathrm{Pr}$ is less than the former, and $G$ is important for the perturbation. The shape of perturbation field highly depends on the heat transfer. It can be seen from (2.23) that the change of $U_{0}$ by gravity can lead to a increasing of horizontal convection $U_{0} \frac{\partial T}{\partial x}$. The perturbation flow field changes significantly at large $\operatorname{Pr}$ as the convection is crucial for the heat transfer. However, it has little effect at small $\mathrm{Pr}$ and the change of perturbation field by gravity is not obvious.

\section{CONCLUSION}

The linear stability analysis is carried out for the thermocapillary liquid layers of Carreau fluid. The parameters of preferred mode are obtained for linear flow and return flow. The effect of gravity is studied. The results show that the shear-thinning effect significantly influences the flow stability, such as the critical parameters, perturbation mode, and the instability mechanism.

For linear flow, which has no viscosity stratification, the flow only yields an anisotropic disturbance stress tensor. The shear-thinning effect leads to a destabilization at small and moderate $\operatorname{Pr}_{e}$ while the stability is increased slightly at large
$\operatorname{Pr}_{e}$. Comparing with Newtonian fluid, the propagation angle of oblique wave increases for $n=0.7$. However, the case for $n=0.3$ is opposite, and the preferred modes changes from upstream to downstream.

For return flow, the viscosity stratification makes perturbation kinetic energy concentrate near the surface. The flow is stabilized when the surface viscosity is used for the definition of Marangoni number and Prandtl number. The preferred mode becomes the streamwise wave at large $\mathrm{Pr}$, while the propagation angle of oblique wave increases at small $\mathrm{Pr}$. The temperature perturbation at moderate $\operatorname{Pr}$ has hot spots at the bottom, which absorb energy from the horizontal convection and heat the interface by conduction. Energy analysis shows that the work done by the perturbation stress $\tau_{13}$ is increased significantly by shear-thinning effect for most of $\operatorname{Pr}$.

In the presence of gravity, the vertical gradients of velocity and temperature in basic flow increase and more kinds of preferred modes are excited. For large $\mathrm{Pr}$, the work done by gravity is negligible for the perturbation energy. The preferred mode changes from upstream to downstream, while the streamlines near the hot spot changes from counterclockwise to clockwise. However, for small $\mathrm{Pr}$, the gravity becomes an important perturbation energy source.

\section{ACKNOWLEDGMENTS}

This work has been supported by the National Science Foundation of China (Nos. 11402271 and 11532015) and sponsored by K. C. Wong Magna Fund in Ningbo University.

\section{APPENDIX: THE DERIVATION OF BASIC FLOWS}

The form of basic flow (2.9) is substituted into the momentum equation (2.5). Then the following equations are derived:

$$
\begin{aligned}
& -\frac{\partial p}{\partial x}+\frac{\partial \tau_{13}}{\partial z}=0 \\
& -\frac{\partial p}{\partial z}+\frac{\partial \tau_{13}}{\partial x}+B o \cdot T=0
\end{aligned}
$$

As the velocity is assumed to be a function of $z$,

$$
\boldsymbol{\tau}=\boldsymbol{\tau}(z), \frac{\partial \tau_{13}}{\partial x}=0, \frac{\partial^{2} p}{\partial x \partial z}=-B o=\frac{\partial^{2} \tau_{13}}{\partial z^{2}} .
$$

Therefore,

$$
\tau_{13}=-\frac{B o}{2} z^{2}+C_{1} z+C_{2} .
$$

For linear flow, $\mathbf{u}=(z, 0,0)$, thus $\tau_{13}$ is homogeneous in $z$, which can only be satisfied when $B o=0$.

For return flow, the two constants $C_{1}, C_{2}$ can be derived numerically with two conditions: the shear stress $\left.\tau_{13}\right|_{z=1}=1$ and the return flow condition (2.11). Then, the strain-rate and velocity of basic flow can be obtained numerically. Substituting (2.9) into (2.6), the temperature of basic flow must satisfy

$$
-U_{0}(z)=\frac{1}{M a} D^{2} T_{b}(z) .
$$

This equation can be solved with the boundary conditions of temperature. 
${ }^{1}$ Crystal Growth Processes Based on Capillarity: Czochralski, Floating Zone, Shaping and Crucible Techniques, edited by T. Dufar (John Wiley \& Sons, 2010)

${ }^{2}$ T. DebRoy and S. A. David, "Physical processes in fusion welding," Rev. Mod. Phys. 67(1), 85-112 (1995).

${ }^{3}$ S. H. Davis, "Thermocapillary instabilities," Annu. Rev. Fluid Mech. 19(1), 403-435 (1987).

${ }^{4}$ M. F. Schatz and G. P. Neitzel, "Experiments on thermocapillary instabilities," Annu. Rev. Fluid Mech. 33(1), 93-127 (2001).

${ }^{5}$ E. B. Guto, E. D. Cohen, and G. I. Kheboian, Coating and Drying Defects (Wiley, New York, 1995).

${ }^{6}$ W. R. Hu and N. Imaishi, "Thermocapillary flow in an annular liquid layer painted on a moving fiber," Int. J. Heat Mass Transfer 43(24), 4457-4466 (2000).

${ }^{7}$ J. J. Chen and J. D. Lin, "Thermocapillary effect on drying of a polymer solution under non-uniform radiant heating," Int. J. Heat Mass Transfer 43(12), 2155-2175 (2000).

${ }^{8} \mathrm{~L}$. Weh, "Surface structures in thin polymer layers caused by coupling of diffusion-controlled Marangoni instability and local horizontal temperature gradient,” Macromol. Mater. Eng. 290(10), 976-986 (2005).

${ }^{9}$ G. Toussaint, H. Bodiguel, F. Doumenc, B. Guerrier, and C. Allain, "Experimental characterization of buoyancy- and surface tension-driven convection during the drying of a polymer solution," Int. J. Heat Mass Transfer 51(17), 4228-4237 (2008).

${ }^{10}$ J. P. Singer, "Thermocapillary approaches to the deliberate patterning of polymers," J. Polym. Sci., Part B: Polym. Phys. (published online).

${ }^{11}$ A. A. Darhuber, J. M. Davis, S. M. Troian, and W. W. Reisner, "Thermocapillary actuation of liquid flow on chemically patterned surfaces," Phys. Fluids 15(5), 1295-1304 (2003).

${ }^{12}$ J. R. Felts, S. Somnath, R. H. Ewoldt, and W. P. King, "Nanometer-scale flow of molten polyethylene from a heated atomic force microscope tip," Nanotechnology 23(21), 215301 (2012).

${ }^{13}$ O. A. Basaran, H. Gao, and P. P. Bhat, "Nonstandard inkjets," Annu. Rev. Fluid Mech. 45, 85-113 (2013).

${ }^{14}$ J. P. Downey and J. A. Pojman, Polymer Research in Microgravity: Polymerization and Processing (American Chemical Society, Washington, DC, 2001).

${ }^{15}$ L. A. Davalos-Orozco and A. E. Chavez, "Thermocapillary convection in a viscoelastic fluid layer under a horizontal temperature gradient," J. Appl. Polym. Sci. 49(0), 141-153 (1991).

${ }^{16} \mathrm{P}$. N. Kaloni and J. X. Lou, "On the convective stability of Oldroyd B fluid subject to a horizontal temperature gradient," in ASME/JSME 2003 4th Joint Fluids Summer Engineering Conference, Honolulu, Hawaii, USA, 6-10 July 2003 (American Society of Mechanical Engineers, 2003), pp. 1601-1606.
${ }^{17}$ K. X. Hu, M. He, and Q. S. Chen, "Instability of thermocapillary liquid layers for Oldroyd-B fluid," Phys. Fluids 28(3), 033105 (2016).

${ }^{18}$ M. Naimi, M. Hasnaoui, and J. K. Platten, "Marangoni convection of nonNewtonian power law fluids in a shallow rectangular cavity," Eng. Comput. 17(6), 638-668 (2000).

${ }^{19} \mathrm{Z}$. Alloui and P. Vasseur, "Onset of Marangoni convection and multiple solutions in a power-law fluid layer under a zero gravity environment," Int. J. Heat Mass Transfer 58(1), 43-52 (2013).

${ }^{20} \mathrm{C}$. H. Chen, "Marangoni effects on forced convection of power-law liquids in a thin film over a stretching surface," Phys. Lett. A 370(1), 51-57 (2007).

${ }^{21}$ Y. Renardy, "Viscosity and density stratification in vertical Poiseuille flow," Phys. Fluids 30(6), 1638-1648 (1987).

${ }^{22}$ R. Govindarajan and K. C. Sahu, "Instabilities in viscosity-stratified flow," Annu. Rev. Fluid Mech. 46, 331-353 (2014).

${ }^{23}$ C. Nouar, A. Bottaro, and J. P. Brancher, "Delaying transition to turbulence in channel flow: Revisiting the stability of shear-thinning fluids," J. Fluid Mech. 592, 177-194 (2007)

${ }^{24}$ R. Liu and Q. S. Liu, "Non-modal instability in plane Couette flow of a power-law fluid," J. Fluid Mech. 676, 145-171 (2011).

${ }^{25}$ C. Nouar and I. Frigaard, "Stability of plane Couette-Poiseuille flow of shear-thinning fluid," Phys. Fluids 21(6), 064104 (2009).

${ }^{26}$ M. K. Smith and S. H. Davis, "Instabilities of dynamic thermocapillary liquid layers. Part 1. Convective instabilities," J. Fluid Mech. 132, 119-144 (1983).

${ }^{27}$ R. J. Riley and G. P. Neitzel, "Instability of thermocapillary-buoyancy convection in shallow layers. Part 1 . Characterization of steady and oscillatory instabilities," J. Fluid Mech. 359, 143 (1998).

${ }^{28}$ Y. R. Li, N. Imaishi, T. Azami, and T. Hibiya, "Three-dimensional oscillatory flow in a thin annular pool of silicon melt," J. Cryst. Growth 260, 28 (2004).

${ }^{29}$ P. J. Carreau, "Rheological equations from molecular network theories," Trans. Soc. Rheol. 16(1), 99-127 (1972).

${ }^{30}$ R. B. Bird, R. C. Armstrong, and O. Hassager, Dynamics of Polymer Liquids. Fluid Dynamics (Wiley/Interscience, New York, 1987), Vol. 1.

${ }^{31}$ P. J. Schmid and D. S. Henningson, Stability and Transition in Shear Flows (Springer, 2001).

${ }^{32}$ M. K. Smith, "Instability mechanisms in dynamic thermocapillary liquid layers," Phys. Fluids 29(10), 3182-3186 (1986).

${ }^{33}$ K. X. Hu, J. Peng, and K. Q. Zhu, "The linear stability of plane Poiseuille flow of Burgers fluid at very low Reynolds numbers," J. Non-Newtonian Fluid Mech. 167-168, 87-94 (2012).

${ }^{34}$ M. Wanschura, V. M. Shevtsova, H. C. Kuhlmann, and H. J. Rath, "Convective instability mechanisms in thermocapillary liquid bridges," Phys. Fluids 7(5), 912-925 (1995). 